\title{
Ellipticity, Its Origin and Progression in Comoving Galaxies
}

\author{
Conrad Ranzan \\ DSSU Research Center, Niagara Falls, Ontario, Canada
}

\section{Email address:}

ranzan@cellularuniverse.org

\section{To cite this article:}

Conrad Ranzan. Ellipticity, Its Origin and Progression in Comoving Galaxies. American Journal of Astronomy and Astrophysics. Vol. 3, No. 2, 2015, pp. 12-25. doi: 10.11648/j.ajaa.20150302.11

\begin{abstract}
Conventional cosmology is based on (i) the Edwin Hubble discovery of the relationship between redshifted light and cosmic distance; (ii) the fact that "space" expansion causes spectral redshifting; and (iii) an extrapolation of space-medium expansion encompassing the entire universe. It is a cosmology that utterly fails in explaining the long-standing mystery of Ellipticals. Within an expanding universe (even an accelerating expanding universe) ellipticity cannot be explained. The present Paper turns to an intrinsically cellular cosmology, one that is based on (i) the same discovery made by Edwin Hubble of the redshifted-cosmic-distance relationship; (ii) the same fact that medium expansion causes photons to stretch; and (iii) the new finding that redshifting can occur in regions where the space medium expands as well as where it contracts; this new development is called the velocity differential theory of cosmic redshift and leads inescapably to inherent cellularity. It is shown, herein, how the universe's cellularity, with its dual-dynamic space medium, is essential for explaining the formation and ellipticity of galaxies (as well as the potential for subsequent galactic interaction).
\end{abstract}

Keywords: Elliptical Galaxies, Comoving Galaxies, Gravity Domain, Gravity Cells, Dynamic Aether, Cellular Cosmology, DSSU

\section{Introduction}

That which cannot be predicted cannot be understood, and therefore, cannot be explained. -apodictic assertion

Without any doubt, there can be no plausible explanation for elliptical galaxies - no explanation for their characteristic features, no explanation for their evolution - within an expanding-type universe model. Within this model-type restriction, the search for understanding has long been and will always be a futile endeavor.

From the time of Edwin Hubble's monumental discovery ${ }^{1}$ [1] of the fundamental relationship between the degree of galactic redshift on the one hand and cosmic distance on the other, Cosmology, the science of the universe, adopted the belief that the whole universe was expanding. Some of the most brilliant scholars of the modern age came to believe the cosmos was expanding explosively; others came to believe it was expanding leisurely and steadily. The belief was based on

\footnotetext{
1 Praise and credit for contributing to the discovery of the cosmic redshift, historically called the astronomic redshift, also goes to several other astronomers including Vesto M. Slipher, the German Carl W. Wirtz, and the Swede Knut Lundmark; as well as the American cosmologist Howard P. Robertson.
}

a grossly unscientific extrapolation and dominated astrophysics research for the rest of the century. From the 1920 s through to the first decade of the present century, the causal mechanism behind Elliptical galaxies (structures as herein defined) was a complete mystery; and under the old cosmology it remains so. According to Andrey Kravtsov, a professor in astronomy \& astrophysics at the University of Chicago, the formation of elliptical galaxies (as well as spirals) is one of the biggest remaining questions in astrophysics [2]. Long time promoter of the old cosmology Stephen Weinberg writes [3], "No one knows how galaxies formed ..." Another such promoter is Roger Penrose, who writes [4], "Here we run into the unresolved and controversial issue of how galaxies are actually formed, ..." Astrophysics textbooks generally avoid the topic; none contain plausible answers. Authoritative encyclopedic sources admit to a major gap in our knowledge, "... the lack of a simple convincing picture of how galaxies form and cluster will remain one of the prime failings of [conventional expansion cosmology]." [5]

Clearly, the explanation for elliptical galaxies, those non-rotating most featureless of all astronomical objects, cannot be had within the expanding universe paradigm. 
During the last several years a number of published research papers have successfully demonstrated that the universe, contrary to Academia's long-held view, is not expanding. Furthermore, the new-cosmology argument includes incontrovertible proof that our Cosmos is intrinsically cellularly structured, contrary to the view of mere phenomenological cellularity.

A remarkable 2009 paper [6] entitled, "The Story of Gravity and Lambda - How the Theory of Heraclitus Solved the Dark Matter Mystery," convincingly argues that hypothetical mystery matter (alias dark matter) is entirely unnecessary within a stably cellularly-structure universe.

The paper [7] "The Processes of Gravitation - The Cause and Mechanism of Gravitation," with its detailed account of Primary, Secondary, and Tertiary gravity, provides a solid theoretical foundation for the cosmic cellular structure.

The most recent published work [8] convincingly demonstrates that spectral redshifting occurs in regions of expanding space (as is well-known and universally accepted) as well as in regions of contracting space. This is an amazing and wholly unexpected development! The mechanism is superior to all previously invoked causes for the cosmic redshift, namely: (i) Doppler shift, (ii) tired or fatigued light, (iii) gravitational or Einstein shift, and, of course, (iv) universal space-medium expansion. The new $5^{\text {th }}$ cause of cosmic redshift, with its intimate connection to cosmic cellularity, makes it crystal clear that there is absolutely no need for the universe to expand. This published revolutionary document removes the main pillar propping up the expanding-universe paradigm.

In another recently published work Guide to the Construction of the Natural Universe [9], it is argued that instead of a one-time creation/formation scenario the universe manifests on the basis of continuous creation/formation in accordance with the principle of limited existence whereby entities neither exist forever nor are recycled - the necessary and self-evident result is a perpetual steady-state cellular cosmos.

And then of course, there are the undeniable observations. Astronomers are routinely reporting the amazing systematic nature of the universe's large-scale structure -its grand cellularity - while, ironically and invariably, complaining that no theory predicts such observed regularity. (It's a perennial story that goes all the way back to the 1960s and 1970s and the pioneering work of French-born American astronomer Gérard de Vaucouleurs and Estonian astronomer Jaan Einasto.)

For the answer to galaxy ellipticity we turn to the cellular cosmology known as the Dynamic Steady State Universe, $\mathrm{DSSU}^{2}$ for short. The following discussion expands on the

\footnotetext{
${ }^{2}$ Dynamic Steady State Universe (DSSU) is the cosmology theory, based on a dynamic aether space-medium, in which aether continuously expands and contracts regionally and equally thereby sustaining a cosmic-scale cellular structure. It models the real world on the premise that all things are processes. Historically, it is the first true Steady State (SS) universe - SS nonexpanding, SS cellular, SS infinite, SS perpetual.
}

success of DSSU cosmology which began at the 2002 ESO-CERN-ESA International Symposium at Munich, Germany [10].

The focus will be on the cause of ellipticity in comoving galaxies and protogalaxies. And to understand the pattern of the comovement we need to understand the nature of the cosmic cell structure.

\section{Voronoi Cell Structure}

\subsection{Cell Shape}

The DSSU is essentially structured as Voronoi cells on a cosmic scale. Each cell has a vast central region (commonly called a void) in which the universal space medium is involved in a process of expansion; it is a region of space-medium expansion. Each cell is enclosed by a shared "boundary" region in which the space medium is involved in a counter process of contraction. There is stability in the size of the cells because the quantity of space medium "produced" in the void region is balanced by the quantity "consumed" (contracted) in the boundary region. These two dynamic regions determine the cell shape. The negative pressure (equivalent to Einstein's positive-value cosmological constant) of the void causes each cell to maximize its volume. To be more precise, when the force involved is that of "negative pressure" then the tendency is to maximize the distance between neighboring cell centers and simultaneously minimize the number of boundary surfaces. Of the three polyhedra cells capable of no-gaps repetitive total tessellation of a 3-dimensional volume, only one fulfills the necessary requirements. The three tessellation candidates are the hexahedron, the truncated octahedron, and the rhombic dodecahedron. The hexahedron has the least number of boundary surfaces (six) but fails when it comes to maximizing the cell-center separation distance. The truncated octahedron is excellent for maximizing the separation between cell centers but has the most number of boundary surfaces (with fourteen). The rhombic dodecahedron maximizes the cell-center separation and has only twelve boundary surfaces.

The idealized shape of the DSSU cosmic cells is the rhombic dodecahedron -a figure with twelve regular rhombus faces (see Fig. 1a). What may otherwise be imagined as a stable sphere enclosing two balancing space-dynamic regions actually manifests as a non-Platonic, quasi-regular, dodecahedron when "close-packed" as part of an infinite universe.

As for the size of these cosmic structures, they are in agreement with astronomical observations [11], viz, approximately 350 million lightyears (Mly) in diameter.

Turning our attention to the cell's largest region, the vast central domain in which the space medium expands: The rate of the expansion is approximately $3.2 \mathrm{~cm} / \mathrm{km}$ per million years (Myr). Later, we will see how this value was derived. To get a feel of this expansion rate, think of it in these terms: Two comoving points, or objects, that are one kilometer apart within the medium will increase their separation by 3.2 
centimeters during one million years of Earth time. This growth of the medium is isometric; one kilometer of the medium, regardless of the direction, expands by $3.2 \mathrm{~cm}$ each and every million years. The universal space medium has a name; we call it the DSSU aether, but note carefully that this aether is comprised of discrete entities that do not possess mass and do not represent energy. The significance of the uniform and isometric nature of expansion is that comoving objects therein do not change shape. Comoving objects will increase their separation from one another but the pattern, or shape, of configuration remains unchanged; comoving structures may spread and grow in size but the shape remains constant. The nature of the expansion is said to be homologous.

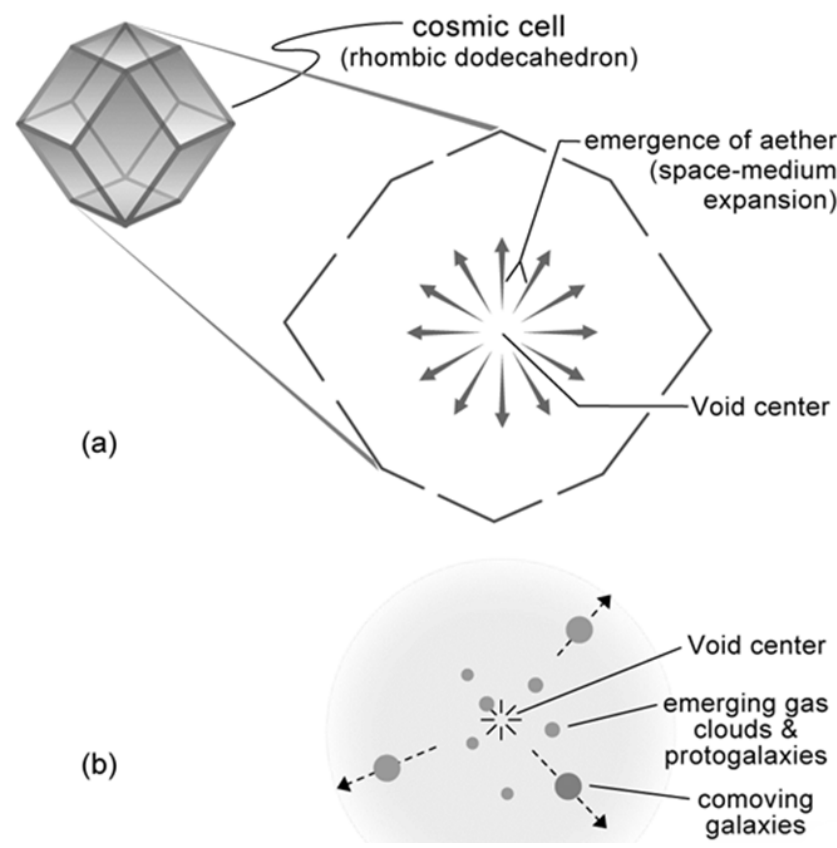

Fig. 1. The voids within cosmic cells are regions of space-medium expansion and tranquil matter formation/precipitation. Part (a) is a schematic of the slow emergence (expansion) of the space medium. But note, the cosmic cell itself does not expand. Part (b) shows the formation of gas clouds, protogalaxies, and galaxies. Matter precipitates from aether and feeds the formation-creation of hydrogen clouds and full-status galaxies. As these structures drift with the dynamic aether, they undergo homologous growth and expansion. They remain spherical.

According to DSSU cosmology the center of voids - being, as they are, by far the most undisturbed regions of the universe - are a nursery for the formation of protogalaxies and even full-status galaxies. Matter precipitates from the aether and forms hydrogen clouds, which in time grow into protogalaxies (structures of incipient and early-stage star formation). In a timeless and ongoing sequence, matter, in its most fundamental form, precipitates from the aether; and simple atoms precipitate from the fundamental matter to form clouds of hydrogen gas; and protogalaxies "condense" from the collection of hydrogen clouds. These structures may sit there at the eye of the divergence zone, at the Void's very center, for countless of billions of years all the while continuing their slow but relentless growth. However, their location is unstable. These protostructures are balanced on a gentle "hill" of expansion; eventual balance is lost and a structure or a group of structures will slide down the expansion hill, so to speak. They will then drift away from the Void center. They will accelerate in comovement with the aether along one or another radial trajectory, as shown in Fig. 1b. Because the aether expansion has a constant rate, the radial acceleration of the aether is constant. And because aether acceleration is constant, so too is the comoving protogalaxy. Finally, since the protogalaxy is accelerating uniformly, there is no change of shape. It will thus retain its spherical symmetry. Any effects such as self-gravitation and homologous aether expansion will not change the spherical shape.

Ellipticity is introduced only when the structure encounters an increase in the acceleration of comotion. And to understand this aspect we need to consider the distribution of galaxy clusters.

\subsection{Galaxy Cluster Domains}

The cosmic structural cells, because of their dodecahedral shape, have fourteen nodes, which correspond to the fourteen rich galaxy clusters that are predicted to surround each cosmic void. See Fig. 2.

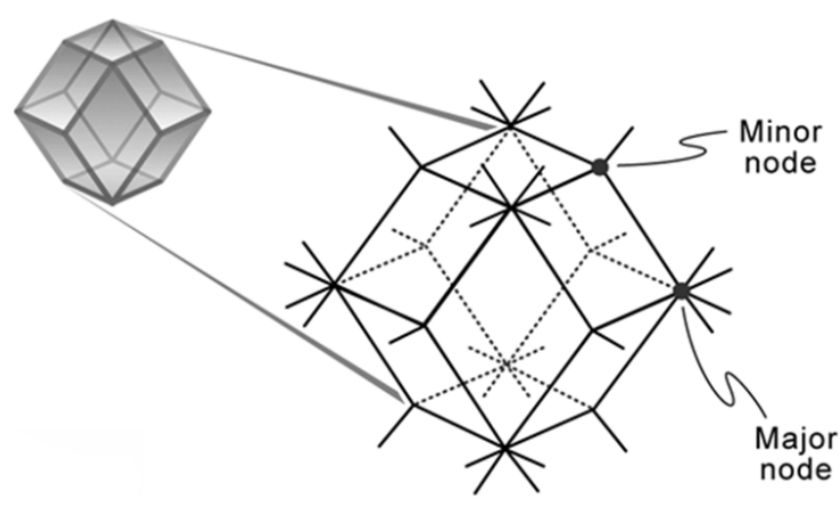

Fig. 2. The fourteen nodes of the externally-linked structural cosmic cell. Nodes are schematically linked outward to the surrounding dodecahedra (not shown) to reveal that Minor nodes actually have four arms and Major nodes eight arms.

It is interesting to note that there are two distinctive types of nodes - distinguished by the number of linking arms. There are major nodes and minor nodes; six of one and eight of the other. The Major nodes have an eight-arm configuration and the Minor nodes have a four-arm configuration giving us a correspondence, respectively, to eight-branch galaxy clusters and four-branch galaxy clusters. We have here the underlying reason for the significant variation in size and density of galaxy clusters.

In the extended dodecahedral tessellation, nodes are always shared with neighboring cosmic cells. Four cells meet at a Minor node and six cells meet at a Major node, resulting in the 4- and 8-branch patterns shown in Fig. 2. Clearly, with the nodes being the centers of gravity, there are two types of gravitational domains. The geometry demands there be two differently shaped gravity domains or cells (one for each of the two types of nodes). 
Consider a Minor-node galaxy cluster, its gravity domain extends into the four neighboring cells; in fact, it extends into the void centers. This three-dimensional domain is defined by those four extreme points. Connect the points together and the result is a tetrahedron. Thus, a Minor galaxy cluster requires a tetrahedral gravity domain. Similarly, consider a Major-node galaxy cluster; its gravity domain extends into the void centers of SIX neighboring cells. The three-dimensional domain that those six extreme points define is an octahedron. Thus, a Major galaxy cluster requires an octahedral gravity domain.

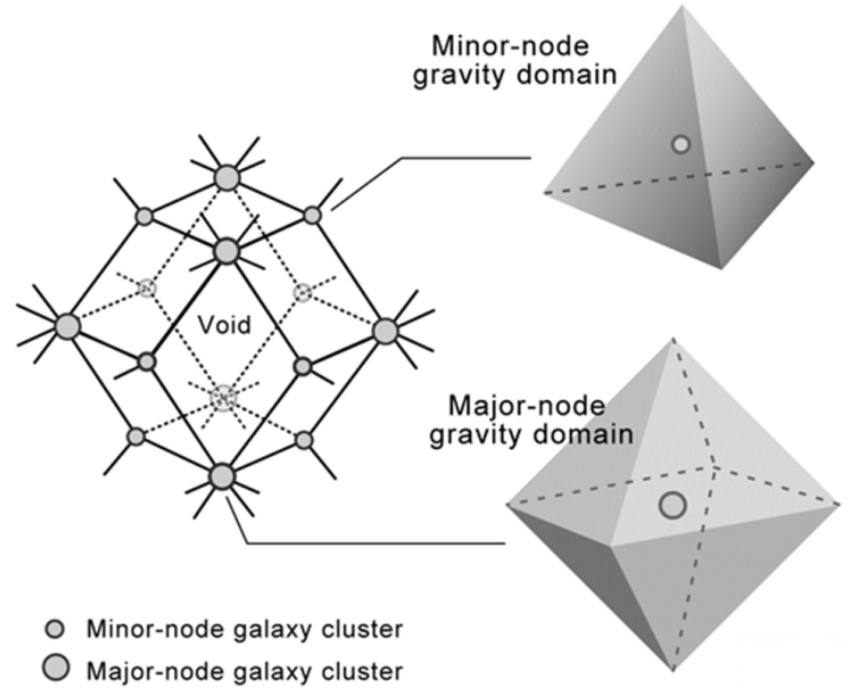

Fig. 3. Cosmic gravity domains. Minor-node galaxy clusters are the centers of tetrahedral gravity domains. Major-node galaxy clusters are the centers of octahedral gravity domains. The tetrahedron has four vertices, each of which corresponds to the void center of one of four neighboring dodecahedra (only one shown). The octahedron has six vertices; each vertex coincides with the void center of one of six neighboring dodecahedra (only one shown).

In terms of the schematic diagram (Fig. 3), the four-armed node is associated with a tetrahedron; while the eight-armed node demands an octahedron. Now, it is a remarkable fact that the tetrahedron and the octahedron combine to form a fully triangulated network. What the rhombic dodecahedron accomplishes on its own, the tetrahedron and the octahedron accomplish in combination. The space-filling geometric property of dodecahedra is matched by a "packing" union of tetrahedra and octahedra. Two tetrahedra and one octahedron combine to form a space-filling parallelipiped [12]. (Observe a counterintuitive aspect here; although an isolated structural cosmic cell is associated with 8 tetrahedra and 6 octahedra, a ratio of 4 to 3 , the actual ratio within an extended array of cosmic cells is simply two to one.)

Figure 4 shows how the 14 domains, associated with one dodecahedral cosmic cell, are packed.

From a structural point of view, the DSSU is a densely packed array of rhombic dodecahedra (as well as trapezoidal-rhombic dodecahedra), which means that they fit together so as to divide up a volume of space without leaving gaps between adjacent cells. Furthermore, from a gravitational point of view, the universe is a packed array of tetrahedral and octahedral gravity domains. This aspect of the universe is nothing more than basic Euclidean spatial geometry. It is the flow of the space medium within these structures that introduces a degree of complexity and regional distorting effects resembling non-Euclidean geometry. However, as I have already pointed out, the cosmic structural cells do not expand and the cosmic gravity cells do not contract. Therefore, the framework of the universe remains Euclidean.

Let us examine in more detail the two kinds of gravity domains, which, when "packed" together as gravity cells, will totally fill 3-dimensional space. It should be emphasized, unlike the cosmic structural cells with their mass-sprinkled envelope of long filaments of galaxies, the gravitation cells have no visible boundaries!

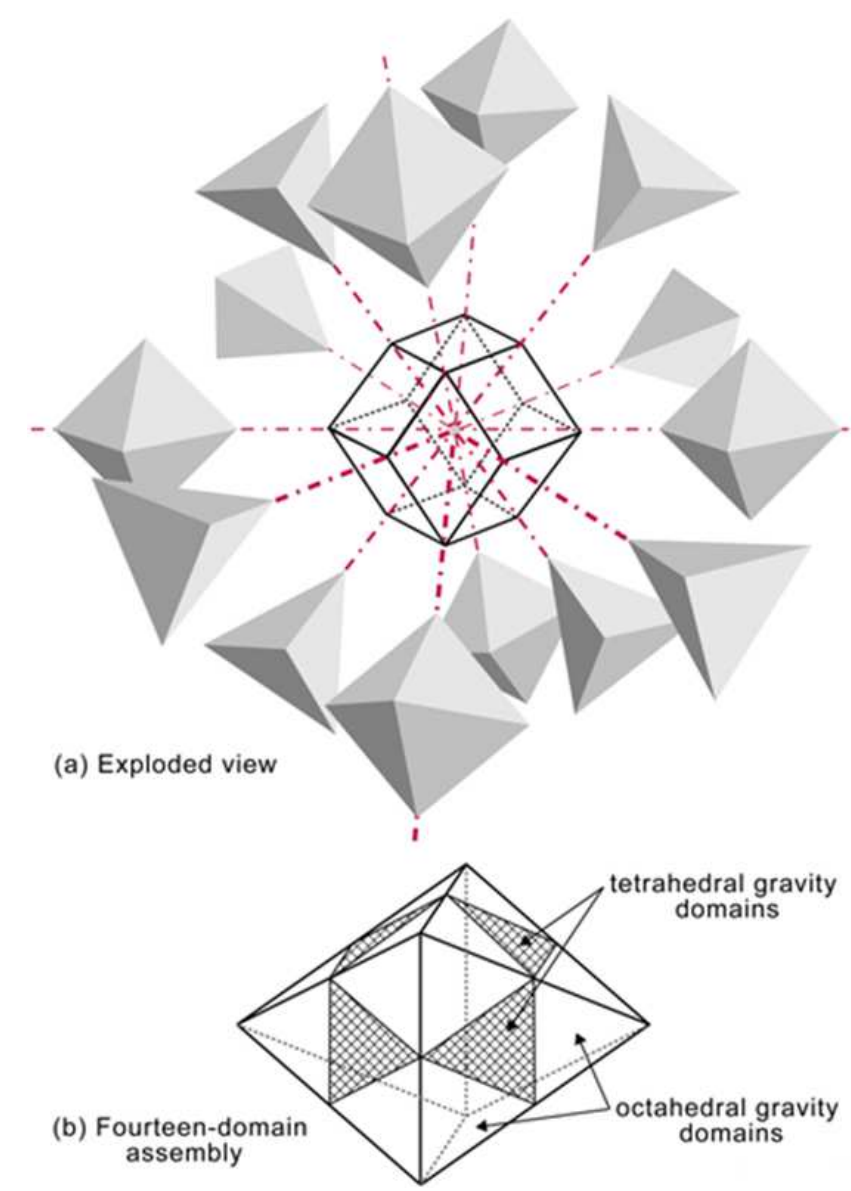

Fig. 4. A "packing" of the 14 gravity domains or cells associated with a dodecahedral structural cell. All 14 domains have a common vertex in the very center. We normally think of the dodecahedral cell as being surrounded by twelve others, each of the twelve faces shared with a different neighbor. However, the actual linking involves a total of eighteen others. A count of the number of boundary vertices, of the super-octahedron including those vertices located at the long-edge midpoints (and including 5 hidden ones) gives a total of 18 void centers - one for each of the 18 "neighbors." (In the exploded view (a), the size relationships and orientations are only approximate.) The complete assembly is in the form of a super octahedron, whose approximate orientation is that shown in part (b). 


\section{Tetrahedral and Octahedral Gravity Domains}

\subsection{Tetrahedral Domain}

The center of gravity of a Minor-node galaxy cluster coincides with the geometric center of the tetrahedral cell (Fig. 5a). The vertices of the tetrahedron are located at the centers of four surrounding Voids. Let us take two of the vertices, push them down, and form a quasi-cross-section as in Fig. 5b. Notice how the region of aether expansion surrounds the region of aether extinction. Most revealing is the pattern of comoving trajectories; the tetrahedral domain has four internal sub-domains (Fig. 5c). The pattern is explained by the DSSU's aether theory of gravity [7].

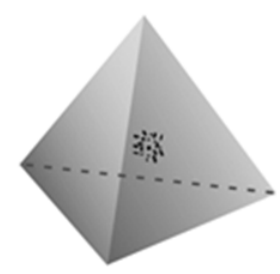

(a) Perspective view (idealized)

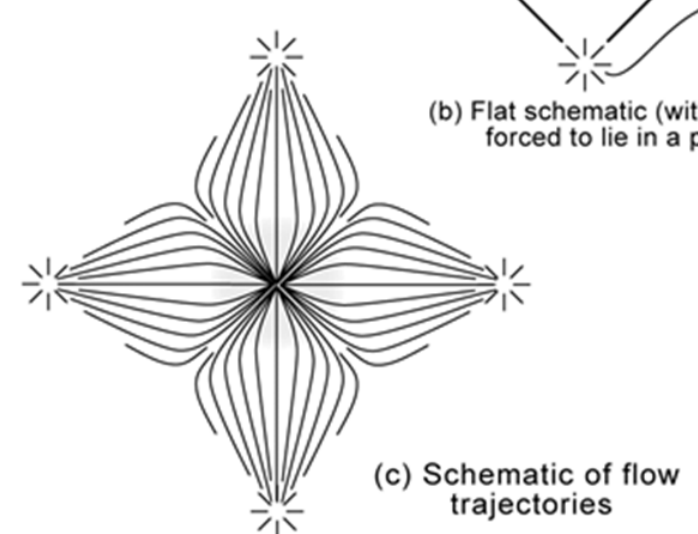

Fig. 5. Anatomy of a tetrahedral gravity domain. (a) Perspective view of the gravity domain surrounding a Minor-node galaxy cluster. (b) Schematic "flat" view showing expansion and contraction zones. Although there is a regional expansion and contraction of the space medium, the gravity "cell" itself remains stable in size - a consequence of its sustaining steady-state processes (c) Schematic of aether-flow trajectories.

\subsection{Octahedral Domain}

Major-node galaxy clusters are the centers of octahedral gravity domains (Fig. 6a). Each vertex of such octahedron is located at the centers of an adjacent Void. If we purposely deform the octahedron to show these Void centers schematically in a plane, we would have a hexagon with the galaxy cluster at the center (Fig. 6b). The anatomy of the domain is shown in Fig. $6 \mathrm{~b}$ and includes the aether-expansion zone, the aether-contraction zone, and the material-aggregation region. The aether flow lines and comoving trajectories are patterned as six sub-domains (Fig. 6c). In the largest region, comotion is divergent (and deformation is homologous); in the mid region, comotion is steadily convergent (and deformation is systematic); in the central region, the region of material aggregation, comotion becomes chaotic as numerous local gravity domains, inside the cluster, overlap and conflict (and deformation is haphazard).

Always keep in mind, the gravity domains (and their sub-domains) do not change in size. The tetrahedral and the octahedral cells do not expand or contract. They are perpetually self-sustaining. The grand web-like (cellular) network of galaxy clusters and superclusters is intrinsically stable.

During their long journey beginning at the extremities of the gravity domain, protogalaxies (and their descendent forms) are subject to symmetrical deformation in the diverging region and then asymmetrical deformation in the converging region. In order to quantify the deformation effects, we need to construct the profile of the aether flow within a gravity domain. We need the graph of the comoving velocity.

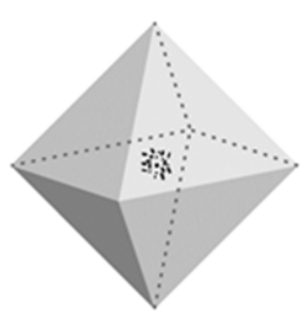

(a) Perspective view (idealized)

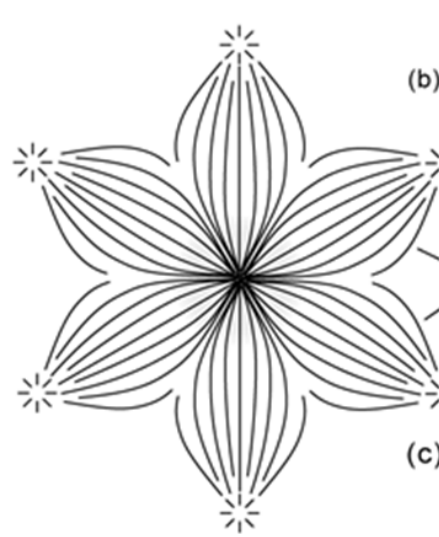

(c) Schematic of flow trajectories
Fig. 6. Octahedral gravity cell is a dynamic structure in which the space medium flows in a pattern that sustains six subdomains - a configuration that is associated with the dodecahedral Major nodes. Part (a) gives a perspective view. In part (b), the octahedron has been flattened out to illustrate the three key regions - namely, the cluster, the main contractile zone, and the Void region. Part (c) shows the approximate pattern of the space-medium flow originating from the six Void centers and ending at the central galaxy cluster. The flow lines are diverging where the space medium expands and converging where the medium contracts.

\subsection{Graph of Comoving Velocity}

First we need the distance - the distance between the center of the galaxy cluster and the farthest extent of its influence. For the tetrahedral gravity domain this distance is 159 million lightyears (Mly); for the octahedral gravity domain it is 184 
Mly. These values are based on dodecahedral cosmic cells having a nominal diameter of $350 \mathrm{Mly}$, in agreement with observations, as mentioned earlier. But there are some geometric details we need to take into consideration. It turns out that a rhombic dodecahedron has three significant diameters. The smallest diameter defines the inscribed sphere that "touches" all the faces of the dodecahedron; the next larger defines the inner circumscribed sphere which passes through all the Minor nodes; the largest diameter defines the outer circumscribed sphere which passes through all the Major nodes. The most accommodating dodecahedron is one with an inscribed-sphere diameter of 260 Mly; the corresponding inner circumscribed sphere then is $318 \mathrm{Mly}$; and the corresponding outer circumscribed sphere has to be 368 Mly. (Notice that the average of the latter two is reasonable close to the deemed nominal diameter of $350 \mathrm{Mly}$.)

What all this means is that the distance of a Minor-node cluster from a Void center is $159 \mathrm{Mly}$; and the distance of a Major-node cluster from a Void center is 184 Mly. And for graph construction, the maximum "radius" of the tetrahedral gravity domain is $159 \mathrm{Mly}$; and the maximum "radius" of the octahedral domain is 184 Mly.

Next we need the size of the respective galaxy clusters.

The mass of galaxy clusters is notoriously difficult to determine. For one thing, they are not spherical but rather have extended arms. For another, astronomers believe there is far more dark stuff than there is visible matter. There is also the already-discussed variation in the configuration of nodes of the dodecahedral geometry arising from 4 arms and 8 arms, all of ideally equal length. But there is a further complication caused by trapezoid-dodecahedral geometry in which linking arms are not of equal length (4-arm nodes with one arm shorter than the others, 8 -arm nodes, with some arms longer than the others).

Astronomers report that a typical galaxy cluster has the mass of 1,000 trillion Suns $\left(10^{15}\right.$ Suns $)$. The generally accepted range of mass values is from about $1 \times 10^{15}$ to $3 \times 10^{15}$ Solar masses. Our nearest galaxy cluster, the Virgo cluster, is believed to have a mass of around $1 \times 10^{15}$ Solar masses [13]. Recently, a team of astronomers calculated the mass of a distant cluster known as El Gordo (Spanish for "the fat one") to be as much as 3,000 trillion Suns (or $3 \times 10^{15}$ Solar masses) [14].

It would, therefore, be reasonable to have the mass of the Minor-node cluster equal $1 \times 10^{15}$ Solar masses (or $2 \times 10^{45} \mathrm{~kg}$ ); and to have the mass of the Major-node cluster equal $3 \times 10^{15}$ Solar masses ( or $6 \times 10^{45} \mathrm{~kg}$ ).

Like astronomers who describe "These objects [as] balls of gas in which galaxies are embedded like seeds in a watermelon,"[13] we will make the simplifying assumption that the clusters are spherical. The assumption is made for the convenience of subsequent calculations knowing full well that the clusters are not actually spherical.

It would also be useful to have the diameter of the nominally-spherical cluster. Here we make use of a basic property of the cosmic gravity well. The speed of comoving freefall (of an object originating from some significant distance) is always greatest at or near the "surface" of the gravitating structure. In other words, the speediest galaxies in a galaxy cluster provide the key clue to the cluster's size, its nominal surface. It is not uncommon to find galaxies with significant motion with respect to the cluster's center. Galaxies have been found freefalling, into a cluster, with a speed of $2000 \mathrm{~km} / \mathrm{s}$ and even $3000 \mathrm{~km} / \mathrm{s}$ [15, 16].

If we take the aether-gravity equation (the aether-inflow equation in the Appendix), rearrange the terms so that

$$
r=\frac{2 G M \mathrm{CL}}{v^{2}}
$$

and substitute values for the comoving velocity, the cluster mass, and Newton's gravitational constant (respectively, $v=$ $2000 \mathrm{~km} / \mathrm{s}$; cluster mass $M_{\mathrm{CL}}=1 \times 10^{15}$ Solar masses; $G=$ $6.67 \times 10^{-11} \mathrm{Nm}^{2} / \mathrm{kg}^{2}$ ), and make the appropriate units conversions, we find that the "surface" has a radius of 7 Mly. The nominal surface of the Minor-node galaxy cluster occurs at a radial distance of 7 million light years.

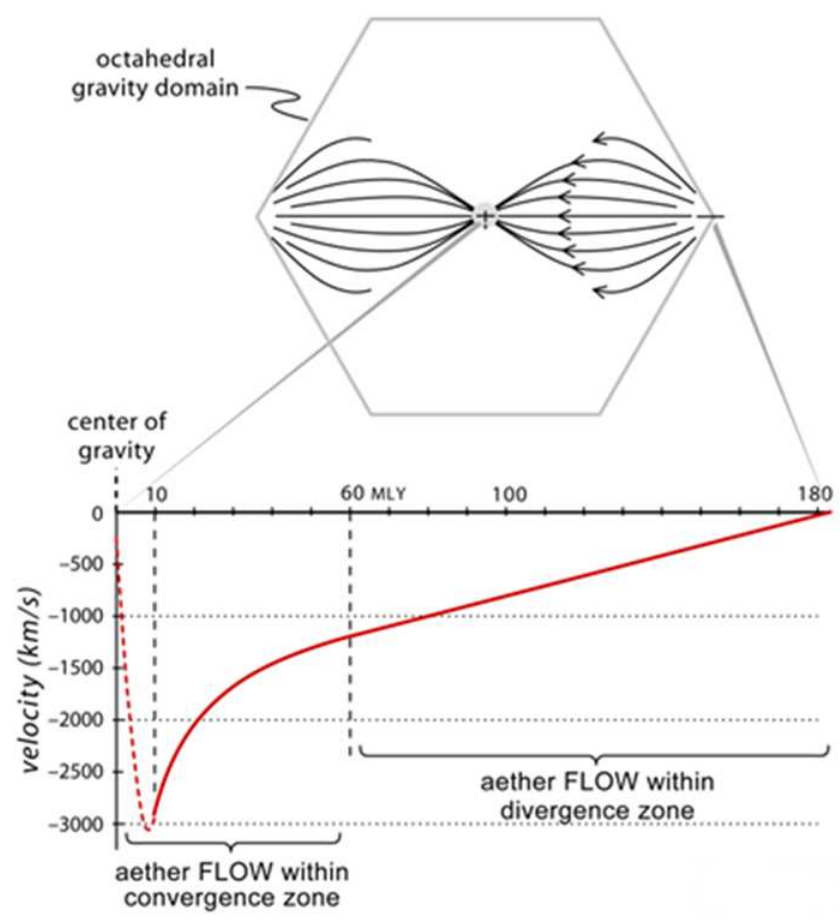

Fig. 7. Graph of comoving inflow for octahedral gravity domain. The profile between $10 \mathrm{Mly}$ (the nominal "surface" of the cluster) and $60 \mathrm{Mly}$ (the outer limit of the contractile region) is a representation of equation (2) using a Major-node cluster mass of $3 \times 10^{15}$ Solar masses. The dashed curve indicates that the comotion goes to zero at some central point of the cluster. In the opposite direction, where profile-axis distance is $184 \mathrm{Mly}$, the velocity is zero -zero by virtue of this being the location of a Void center. (Velocity is negative because it is in the negative direction of $r$.)

If we similarly substitute the comoving velocity of 3000 $\mathrm{km} / \mathrm{s}$ and the cluster mass of $3 \times 10^{15}$ Solar masses, we find that the nominal surface of the Major-node galaxy cluster occurs at a radial distance of 10 Mly.

With the information available, let us now draw the comotion graph for the octahedral gravity domain. The contractile portion of the domain is essentially a graph of the 
aether-gravity flow equation (see Appendix):

$$
v_{\text {aetherflow }}=-\sqrt{2 G M \mathrm{cL} / \mathrm{r}} .
$$

Obviously, the velocity at the center of the void, where $r$ equals 184 Mly in the graph (Fig. 7), must be zero. This is so by definition (a feature of the universe's Euclidean framework). Furthermore, since the expansion in the aether-expansion zone is uniform (homologous), the comotion there must be a linear function. We, therefore, simply draw a straight line from the void center and extend it to meet the cluster-gravity-well curve at a tangent. (Incidentally, the uniform-expansion-motion equation, when expressed with respect to time, is exponential; the motion equation which we have used here, however, is with respect to radial distance, not time, and is linear.) The point where the two curves touch indicates where the Newtonian-type inflow equals the expanded-aether outflow (the outflow from the Void). The point of tangency occurs at a radius of 60 Mly.

Thus, the region of great interest to us, the region in which comotion is convergent and deformation is systematic extends from 10 to $60 \mathrm{Mly}$. It is this zone that influences the ellipticity of comoving structures.

\section{Factors Affecting Ellipticity}

Once a galaxy or protogalaxy leaves the source region and enters the contractile portion of a cosmic gravity well, it is subjected to four distinct effects: The comoving speed differential (a difference in magnitude along the axis of the trajectory); the lateral contraction effect (a difference in the direction-of-motion effect); the lateral aggregation of matter; and self-gravitation.

\subsection{Comoving Velocity Differential}

As a galaxy proceeds along its comoving trajectory (defined by the cosmic gravity well) its leading edge is travelling faster than its trailing end. Consequently, there will be a tendency for the galaxy to increase in length.

The rate of growth in length $\ell$ can be expressed as $d \ell / d t$. Furthermore, the growth rate is proportional to the length itself. In equation form, $(d \ell / d t) \propto \ell$; and introducing a parameter of proportionality we have the growth expression,

$$
\frac{d \ell}{d t}=k \ell
$$

where $k$ is the fractional time-rate-of-change parameter, and can be expressed as

$$
k=\frac{1}{\ell} \frac{d \ell}{d t} .
$$

Now, referring to Fig. 8, the algebraic length of the galaxy is the coordinate of the galaxy's front end minus the coordinate of the galaxy's back end; that is, $\ell$ is equal to $\left(r_{1}-\right.$ $\left.r_{2}\right)<0$. And $d \ell / d t$ is simply the velocity with which the galaxy is expanding along the $r$-axis (coinciding with the longitudinal axis). This expansion velocity is the algebraic difference between the front-end velocity and the back-end velocity; that is, $d \ell / d t$ is $\left(v_{1}-v_{2}\right)<0$. With these substitutions we obtain,

$$
k=\frac{\left(v_{1}-v_{2}\right)}{\left(r_{1}-r_{2}\right)}>0 .
$$

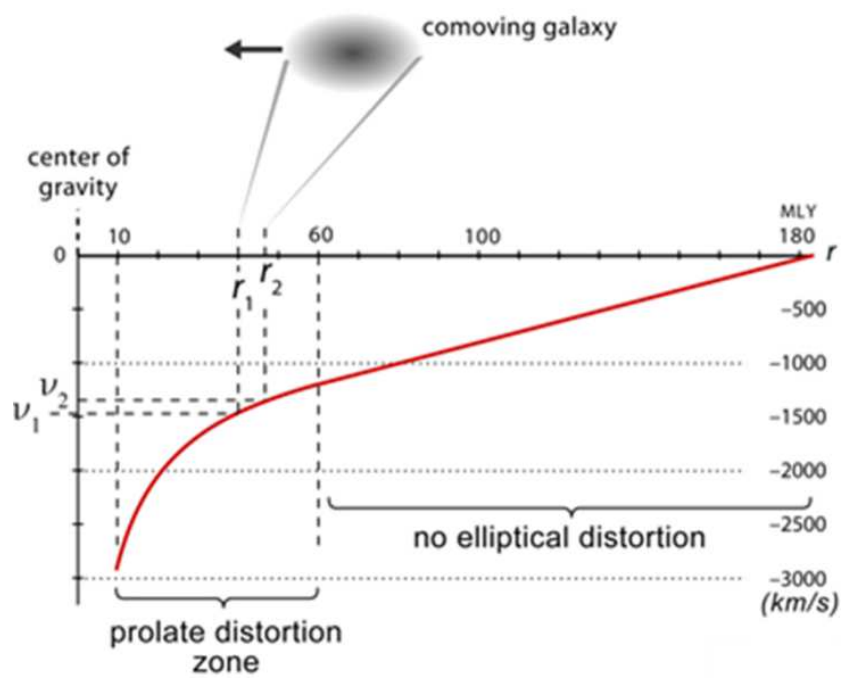

Fig. 8. Velocity differential between front end and back end of any galaxy/protogalaxy structure causes an increase in the axial diameter (axial to the trajectory). The slope to the curve is used to calculate the contribution to ellipticity caused by the velocity effect within the contractile zone (between 10 and 60 million lighyears for the octahedral gravity domain). Note that in the linear portion, the lateral velocity differential is the same as the axial differential, and so, no shape distortion occurs there; this is simply the result of homologous "space" expansion.

By simple inspection, we see that this is just the slope of the aether-flow curve (the comoving velocity (2)) at the location of the galaxy (Fig. 8). Let us generalize the location as $r$. Then the slope at $r$ is

$$
\begin{gathered}
k(r)=\frac{d v_{\mathrm{co}}}{d r}=\frac{d}{d r}\left(-\sqrt{2 G M_{\mathrm{CL}} / r}\right), \\
k(r)=\frac{1}{2} \sqrt{2 G M_{\mathrm{CL}}}\left(r^{-3 / 2}\right) .
\end{gathered}
$$

With the substitution of (6) into (3), the growth expression becomes,

$$
\frac{d \ell}{d t}=\frac{1}{2} \sqrt{2 G M_{\mathrm{CL}}}\left(r^{-3 / 2}\right) \ell,
$$

or equivalently,

$$
d \ell \frac{d r}{d t}=\frac{1}{2} \sqrt{2 G M_{\mathrm{CL}}}\left(r^{-3 / 2}\right) \ell d r .
$$

But $d r / d t$ is just the comoving velocity $v_{\text {co }}=\left(-\sqrt{2 G M_{\mathrm{CL}} / r}\right)$. After making this substitution the equation simplifies to 


$$
\frac{1}{\ell} d \ell=-\frac{1}{2 r} d r
$$

A useful formula for $\ell$ is found by integration (between initial and final quantifiers as indicated by the $i$ and $f$ subscripts):

$$
\begin{gathered}
\int_{\ell_{\mathrm{i}}}^{\ell_{\mathrm{f}}} \frac{1}{\ell} d \ell=-\frac{1}{2} \int_{r_{i}}^{r_{f}} \frac{1}{r} d r . \\
\left.\ln \ell\right|_{\ell_{\mathrm{i}}} ^{\ell_{\mathrm{f}}}=-\left.\frac{1}{2} \ln r\right|_{r_{i}} ^{r_{f}}, \\
\ln \ell_{\mathrm{f}}-\ln \ell_{\mathrm{i}}=-\frac{1}{2}\left(\ln r_{f}-\ln r_{i}\right), \\
\ln \left(\frac{\ell_{\mathrm{f}}}{\ell_{\mathrm{i}}}\right)=\frac{1}{2} \ln \left(\frac{r_{i}}{r_{f}}\right), \\
\frac{\ell_{\mathrm{f}}}{\ell_{\mathrm{i}}}=e^{1 / 2 \ln \left(r_{i r} / r_{f}\right)}, \\
\ell_{\text {final }}=\ell_{\text {initial }} \times \sqrt{\frac{r_{\text {initial }}}{r_{\text {final }}}} .
\end{gathered}
$$

Let us apply the formula to the portion of the gravity well starting where the space-medium contraction begins, at a radius of 60 , and ending where the galaxy cluster nominally begins, at a radius of 10 . With $r_{\text {initial }}$ equal to 60 Mly and $r_{\text {final }}$ equal to $10 \mathrm{Mly}$, we find the "final" length to be

$$
\ell_{\text {final }}=\ell_{\text {initial }} \times \sqrt{\frac{60}{10}}=2.5\left(\ell_{\text {initial }}\right) .
$$

Thus the velocity differential effect induced by a Major cluster will stretch a galaxy by a factor of 2.5; all while comoving a distance of about 50 Mly. And the travel duration? Using the comoving velocity (2) and using care to make appropriate conversion of units, we can find the travel time, which turns out to be 9,400 million years.

A similar calculation for a Minor-node cluster predicts that the velocity differential effect will stretch a galaxy by a factor of 2.7 while comoving a distance of about $42 \mathrm{Mly}$ (from $r=50$ to $r=7)$. The corresponding travel time is 12,600 million years.

Of course, the velocity differential effect is also active in the linear portion of the gravity well (the part between $r=60$ Mly and the Void center at $r=184$ Mly, in Fig. 7 and 8); but there the effect is spherically symmetrical and, therefore, does not induce a change of shape. If anything, it may contribute to the spherical expansion of protogalaxies.

It should be pointed out that, within this linear portion, the comoving velocity is due almost entirely to the process of "space" expansion. It is the rate of expansion that determines the flow velocity - and the rate of expansion is simply the slope of the graph. From Fig. 7 and 8 the slope is $9.5 \mathrm{~km} / \mathrm{s}$ per Mly. This slope, with the appropriate conversion of units, gives the isometric expansion rate of $3.2 \mathrm{~cm} / \mathrm{km}$ per Myr mentioned earlier. The fractional expansion rate is simply 0.000032 per million years.

\subsection{Lateral Contraction Effect}

Let us consider what is happening to the Elliptical in the lateral direction of the in-falling motion. As the Elliptical descends into the gravity well of the cluster, the diameter of the well narrows - the circumference of the well contracts (a direct consequence of aether contraction).

The distance between any two comoving points on the circumference will change in the same proportion as will the circumference itself. For the Major-node gravity well, the circumference at radius $60 \mathrm{Mly}$ is $2 \pi 60 \mathrm{Mly}$; and the circumference at radius 10 Mly is $2 \pi 10$ Mly. The size-reduction ratio is $2 \pi 60: 2 \pi 10$ or 6 to 1 . The circumferential contraction of the well induces the galaxy's lateral axis to contract; if considered in isolation in the assumed absence of other effects, the lateral axis would contract to $1 / 6$ of its initial value.

Now let us apply both lateral and longitudinal effects.

Assume $2 a$ is the initial spherical diameter at the instant that the protogalaxy is about to descend into the curved portion of the well. Upon reaching the bottom of the well 9.4 billion years later, the lateral diameter will be $(1 / 6) \times 2 a$; while the longitudinal diameter will be $(2.5) \times 2 a$ (as calculated earlier in (12)).

Combining the two effects gives the following longitudinal-lateral ratio:

$($ long axis $) /($ lateral axis $)=(2.5 \times 2 a) /((1 / 6) \times 2 a)=15 / 1$.

Needless to say, galaxies with this degree of ellipticity have never been observed - at least not true Ellipticals. (Rotating galaxies, on the other hand, can, and sometimes do, appear extremely flattened and elongated.)

But we are not finished yet. Elliptical structures, during their descent are subjected to another lateral effect.

\subsection{Lateral Aggregation}

During the descent there is a significant amount of lateral accretion of matter - and a corresponding increase in the lateral diameter. Two processes are involved: The formation/creation of matter throughout the Void; and density increase caused by the contraction/self-extinction of aether. The first is a quantitative unknown; the second is easily determined.

Here is how the lateral density increase comes about: We already know the radial comoving velocity is

$$
v_{\mathrm{co}}=\frac{d r}{d t}=-\sqrt{2 G M \mathrm{cL} / r} \text {, }
$$

where $r$ is the radial distance from the center of the cluster mass. Since the circumference, $C$, at $r$ is equal to $2 \pi r$, we can substitute $r=C / 2 \pi$ into the above equation. Then 


$$
\frac{d C}{d t}=-2 \pi \sqrt{2 G M \mathrm{CL} / r} .
$$

It might help to think of the circumference as a comoving shrinking great circle. A comparison of (13) and (14) tells us that the well's circumference is shrinking $2 \pi$ times faster than the position radius (the $r$ location of the galaxy).

The fractional change along the gravity-well circumference at $r$ can be found by dividing (14) by the circumference itself, so that fractional change per unit of time is

$$
\frac{d C}{d t} \frac{1}{C}=\frac{-2 \pi}{2 \pi r} \sqrt{2 G M \mathrm{CL} / r}=-\sqrt{2 G M \mathrm{CL} / r^{3}} .
$$

This means a change in distance between any comoving points on the well great circle is proportional to $-\sqrt{2 G M \mathrm{CL} / r^{3}}$. Also, a change in distance between such points is proportional to the distance itself (the distance along the great circle). Thus, the change-in-separation expression is

$$
v_{\text {lateral }}=-\sqrt{2 G M \mathrm{CL} / r^{3}} \times D_{\text {lateral }} .
$$

It follows that two comoving objects on the same great circle (i.e., on the same circumference of the idealized cluster's gravity well) will have their velocity so defined, with respect to each other. Objects on the great circle tend to move towards each other (this is the meaning conveyed by the negative sign) along the great circle. Since there are countless galaxy-cluster great circle intersecting the comoving Elliptical, then, effectively, material is approaching from all lateral directions.

Be aware, this is not a conventional gravity effect. Notice the direct relationship; the greater the lateral distance, the greater the approach speed. In contrast, with a basic gravity effect, the smaller the distance, the greater is the approach speed. Consider a couple of examples for an Elliptical located midway down the gravity well, say at $r=30$ (for the Major-node cluster). If the lateral distance to some gas clouds is $1 \mathrm{Mly}$, then those clouds would be approaching (because of the contraction of the intervening space medium) at $56 \mathrm{~km} / \mathrm{s}$; if the lateral distance is $10 \mathrm{Mly}$, then the material would be approaching at $560 \mathrm{~km} / \mathrm{s}$; and if $20 \mathrm{Mly}$, then the speed would be $1120 \mathrm{~km} / \mathrm{s}$. Although, such approach is independent of the gravity effect (between Elliptical and the surrounding gas clouds), the fact remains that the closer the gas clouds come to the Elliptical, the more readily they become gravitationally captured and incorporated into the evolving Elliptical structure.

The important point is that material is added in the lateral direction, and hence, comoving Ellipticals grow "wider." No such accumulation occurs in the direction of the longitudinal axis. Along this axis, higher up in the gravity well, hydrogen clouds are moving slower and are therefore moving relatively away from the Elliptical. See Fig. 9. And lower down the longitudinal axis, down the gravity well, the clouds are moving faster and are clearly moving away from the Elliptical.

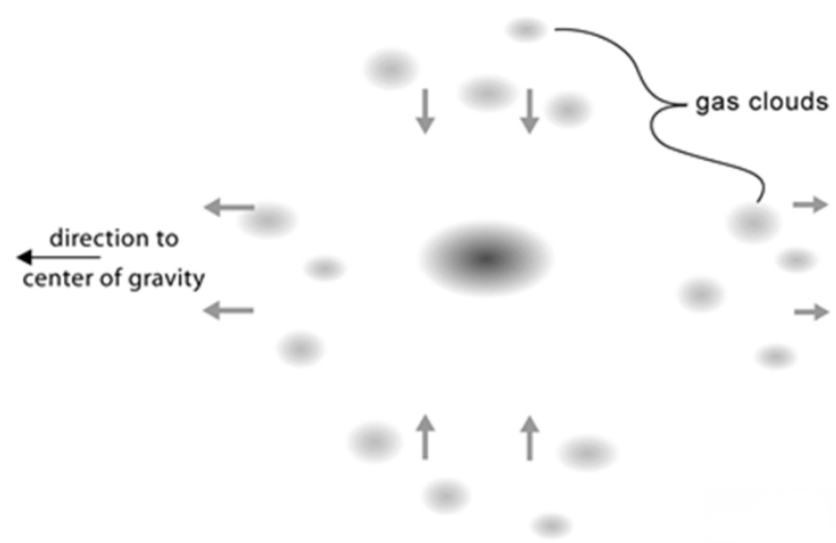

Fig. 9. Relative motion of comoving matter in the vicinity of a galaxy (or protogalaxy) within the contractile region surrounding any nodal galaxy cluster. The pattern of relative motions of hydrogen gas clouds explains galaxy structure growth via lateral aggregation.

\subsection{Self-Gravitation}

The shape of the galaxy is, of course, also influenced by its own contractile gravity. But for the most part, it is of minor consequence.

\subsection{Summary}

The four effects are summarized in Table 1. One effect - the comoving velocity difference between the leading end and the trailing end- stretches the galaxy. Another effect - the lateral contraction - acting somewhat like a tidal distortion squeezes it. And a third - the lateral agglomeration of basic star making matter- fattens it.

Table 1. Summary of shape-changing effects on a galaxy during commotion.

\begin{tabular}{lll}
\hline Effect & Contribution to Ellipticity & Net Effect \\
\hline $\begin{array}{l}\text { Comoving velocity } \\
\text { difference }\end{array}$ & $2.5: 1$ to $2.7: 1$ & \\
Lateral contraction & $6: 1$ & $\frac{(\text { long axis })}{(\text { lateral axis })}=\frac{10}{3}$ \\
Lateral aggregation & significant lateral growth & \\
Self-gravitation & minor & \\
\hline
\end{tabular}

In addition to the formation/creation of matter in otherwise empty regions, there is almost certainly a process of the internal growth of matter. The hypothesis is that protogalaxies grow internally; matter spawns the formation of more matter. By some unknown mechanism, involving aether as always, matter induces the formation of additional matter.

When all the effects are included, the formation/creation of matter both inside and outside the protogalaxy, and the relentless increase in density along the comotion trajectory, and the galaxy's ever-present self-gravitation, the end result is a diameter ratio of 10 to 3.... No Elliptical galaxy has ever been observed more elongated. When a full-treatment Elliptical reaches the "bottom" of the cluster gravity well and astronomers are blessed with an optimal viewing angle, its 10:3 prolate shape will earn it an E7 classification.

Elliptical galaxies are classified according to the degree of 
ellipticity - apparent ellipticity. The categories range from $E 0$, for spherical, then $E 1, E 2$, and so on, through to $E 7$ with the greatest elongation. The $E$-index number is determined by the formula, $10(a-b) / a$; where $a$ is half the long axis and $b$ is half the lateral axis.

After "shape," non-rotation is the essential characteristic. Notice that there is nothing in the described scenario that could possibly cause galaxy rotation. It is a notable observable feature of true Ellipticals that they are not rotating. Aside from the prolate shape, they are the most featureless objects of the universe. As described in the Cambridge Encyclopedia of Astronomy [17], these "galaxies are remarkable for not showing any special features. They look like symmetrical clouds of stars, usually denser in the middle and containing a nucleus, but sometimes without such a condensation. They closely resemble one another, the main variation being the degree of flattening and the rate at which the surface brightness decreases outward."

Eventually, when a galaxy emerges from leisurely comotion it literally tumbles into the waiting cluster. When astronomers detect these galaxies, "they find that they do rotate but with a slow tumbling motion." Moreover, when astronomers compare the rotation speeds of those elliptical galaxies that have actually acquired rotation, they find to their surprise that there is no relation between the speed of rotation and the degree of flatness [18]. It is significant that there is no connection between rotation and elongation. Such observations serve as the crucial evidence that true Ellipticals form-and-evolve without any self-rotation. Rotation is a feature acquired only after a galaxy enters the cluster's urban and suburban environs. Thus, our theory prediction of non-rotation agrees with observations.

\section{Compare-and-Contrast Discussion}

\subsection{Underlying Substrate}

The success of the DSSU theory on the formation and evolution of galaxies is predicated on the existence of aether. The theory fully exploits the existence of an underlying substrate - a discretized essence of reality.

Meanwhile, other schools of thought are just beginning to explore the possibilities. Let me quote one of the experts at University of Cambridge in England, "The possibility exists that we can look for a unified theory of the Standard Model and gravity in terms of an underlying, superfluid substrate of reality." The expert is Ross Anderson, a computer scientist and mathematician; he and his collaborators plan to model their version of aether by using what they call "rotons," particle-like excitations in superfluid helium, as a close analog of this possible "superfluid model of reality." They readily admit the idea is risky (i.e., risky to their careers) and the connections with gravity are speculative. "With physicists it's such a controversial thing, and people are pretty noncommittal at this stage," said one of the risk-averse colleagues. "We're just forging ahead, and time will tell. The truth wins out in the end."[19]
Brian Greene, the grand master of the conventional school, refers to the underlying substrate as the fabric of the Cosmos and the "fundamental framework." (Being career conscious, he shuns the term "aether.") Other than its existence and its discretized nature, he gives precious few characteristics of the aether-like space medium. Quoting Brian Greene, "Physicists believe that, as with water, the smooth portrayal of space and time is an approximation that gives way to another, more fundamental framework when considering the ultramicroscopic scales. What that framework is -what constitutes the "molecules" and "atoms" of space [itself] — is a question currently being pursued with great vigor. It has yet to be resolved."[20]

\subsection{Aether-Based Gravity Theory}

The DSSU is based on an aether-gravity theory that is unique. It is unique in that it does not consider aether to be a physical substance. While all other aether-based "theories" require a space medium consisting of mass particles and /or energy entities, the DSSU conceptualization of aether denies both. Its discrete units possess neither mass and nor energy. Furthermore, DSSU aether has a constant density, that is to say, the spatial density of the discrete aether units is invariant. This represents a radical departure from other aether-gravity models - constructions almost always based on the variation in the medium's density (or the density of some property of the medium).

In a nutshell, DSSU aether [21] is a nonphysical, essence, "substance" - a substance from which all else is derived. It is the ultimate source of all physical phenomena.

Incidentally, it is the premise of constant spacing density that explains the dominant contraction mechanism, which plays such an important role in the shaping (stretching) of elliptical structures. The "contraction of aether" does not mean an increase in density of aether, but rather an extinction (a disappearance) of aether. It is this process, as well as its harmonious opposite, that makes our aether dynamic. In contrast to the DSSU, all other examined theories based on aether invoke a change in density in various attempts to achieve a space medium with dynamic properties.

\subsection{Galaxy Formation}

A major question still plaguing conventional cosmology is: what causes some collapsing gas cloud aggregations to become a spiral galaxy while others collapse to become an elliptical galaxy? Even the variation in size and shape is not understood. Quoting from an astronomy textbook [22], "Why galaxies have such diverse shapes and sizes is a major unsolved puzzle." Inevitably, many theories have been proposed. At one time it was thought that the stars in elliptical galaxies occupy a volume in space described as an oblate spheroid, which is a figure obtained by rotating an ellipse about its minor axis. The amount of pole-to-pole flattening determines the ellipticity [17]. But notice the contrast here; in the DSSU version, Ellipticals are shaped as prolate spheroids, which is a class of figures obtained by 
rotating an ellipse about its major axis. The amount of pole-to-pole elongation determines the ellipticity.

Galaxy formation process: In our DSSU, the formation of galaxies is an ongoing process; the precipitation of new protogalaxies is continuous; the growth of young galaxies is incessant. A never-ending supply of elliptical structures is transported along cosmic conveyor systems and fed into the nodal clusters. The DSSU presents a picture of unceasing assembly lines supplying basic structures for subsequent further modification, cannibalistic consumption, and final disposition.

The continuous-formation process, as a realistic theory, is supported by three compelling pieces of evidence. First, there are the persistent reports of galaxies drifting about within the cosmic voids - even at Void centers. More will be said about this below. Second, there are the decades old observations of numerous small gas clouds dispersed throughout intergalactic space. The observations are based on absorption-line patterns found in the light spectra of distant quasars. The pattern of dark lines is caused by the light passing through cooler intervening gas clouds which capture (absorb) specific tell-tale wavelengths; from the resulting pattern, the presence of hydrogen can be deduced. The third piece of evidence is the detection of protogalaxies. In 1989, astronomers Martha Haynes and Riccardo Giovanelli announced the discovery of an embryonic galaxy described as an enormous cloud (10 times the size of the Milky Way) of hydrogen with a rough elliptical shape, located 65 million lightyears away, within an otherwise empty region of space south of the constellation Virgo. Haynes and Giovanelli used a ground-based radio telescope in the detection of their protogalaxy. In 1995, another team of astronomers using the Hubble Space Telescope, found an additional protogalaxy. As reported in Scientific American [23], the analysis of the spectra collected, by the Space Telescope, from two quasars (separated by a significant angular distance) revealed that a single hydrogen-cloud formation stretched across both lines-of-sight giving it a minimum diameter of one million lightyears. While previous research suggested that intergalactic clouds are about 100,000 lightyears across (about the diameter of the Milky Way galaxy), this one was a comparative monster. Most significantly the cloud "had remarkably little internal motion, suggesting it is a settled structure, not collapsing or flying apart." The researchers involved in the study, not having a workable theory of galaxy formation, were clearly baffled: One wondered, "How can the cloud be so large and so quiescent?" Another commented, "There's no well-developed theory to explain the kind of cloud we see."[23] We, of course, recognize it as a comoving structure, a protogalaxy.

In contrast to our continuous-formation scenario, the conventional view is that galaxies were formed long ago and all at once (and ever since, there has simply been a random process of gravitational aggregation). The problem is that regardless of how far astronomers peer into the distant universe (which they interpret as the early stages of Big Bang evolution), fully-formed mature galaxies (bright and spiral types) are always present. The protogalaxy stage is missing - the evolutionary phase, of the Big Bang, between the universe-as-a-vast-hydrogen-cloud stage and the emergence of protogalaxies that supposedly condensed out of the expanding/exploding primordial ball of gas, is absent. No matter to what depth of redshift distance is explored, astronomers keep finding mature galaxies. In 2013 it was reported that researchers had discovered the most distant examples of galaxies in the early universe, when the universe was supposedly just 1.6 billion years old. These galaxies "were already mature and massive."[24] Then later in the year, astronomers at Texas A\&M University and the University of Texas at Austin announced the discovery of an even more distant galaxy [25]. It was named "z8_GND_5296" and had a spectroscopically confirmed distance of $z=7.51$, corresponding to 700 million years after the Big Bang! ... Bright mature galaxies having formed in such a short time span makes no sense at all. Once again, astronomers are baffled.

\subsection{Relevancy of Evidence}

Under the DSSU worldview, the evidence provides essential support for its galaxy-formation mechanism. The evidence fits the theory's predictions. (Although the present article focuses on the Elliptical stage, the extended theory covers all stages, all morphologies, in good agreement with observational evidence.)

In contrast, under the Big Bang paradigm, the very same evidence is treated as merely phenomenological - the occurrences of a chaotically evolving cosmos. Under a world picture dominated by randomness, it is difficult to the point of being near impossible to devise a plausible model of galaxy formation (and evolution) in keeping with the evidence discussed.

As an example, consider the undeniable evidence [26] of the existence of galaxies in the interior of "voids." The region known as the Pegasus void has an obvious clump of galaxies at its center. A similar situation occurs in the Bootes void. This kind of evidence, since it doesn't fit any standard theory, is generally ignored. Astronomers invariably seem surprised when they find additional instances. A recent Science Daily article [27] is typical. Although "The spaces [voids] in the cosmic web are thought to be staggeringly empty," a team of astronomers based at The University of Western Australia have reported that the voids do, however, exhibit a few galaxies. Using data from the Galaxy and Mass Assembly (GAMA) survey, the team is trying to understand the small population of galaxies that inhabit them.

The evidence of galaxies inside voids (and at void centers) is the evidence of the universe's galaxy nurseries, in agreement with the DSSU prediction. The conventional view treats the same evidence as mere phenomenological features of a cosmos that must be understood in terms of random processes [28]. These galaxies, according to the conventional wisdom, are where they are by the chaotic whim of nature. 


\subsection{Key to Understanding}

In the framework of the DSSU, it is the cellular structure that is the key to understanding galaxy formation and evolution.

In contrast, the conventional claim is that something called dark matter holds the key. In an article written for Sky and Telescope, Astrophysicist G. Bothun of the University of Oregon explains the essential (but misguided) role of hypothesized dark material, "Like most problems in cosmology, until we actually understand the amount and nature of dark matter in the Universe, we will be one step removed from learning the fundamental physics of galaxy formation and evolution."[29]

\section{Profound Implications}

\subsection{Ultimate Source}

An obvious question is where does all the raw material come from? ... Evidently, the growth of protogalaxies and fully-developed galaxies is fed by the hydrogen that precipitates like a fog within the intra- and inter-galactic medium. Small and large clouds of almost pure hydrogen are found widely dispersed throughout the universe. What surprises many is that these clouds seem never to have been associated with any galaxy or cluster of galaxies, which have occasionally been observed to spin off such material. The usual speculation is that they are either transient phenomena or leftovers from the birth of the universe [30]. In our DSSU steady-state cosmos all processes are continuous, hence, the precipitation-formation of hydrogen clouds is an on-going process. But where does the hydrogen come from? The fundamental constituents that make up hydrogen are formed, without violating conservation laws, from the space medium itself - from the essence "substance" that not only moves galaxies but also makes galaxies. Aether provides the raw components. Without going into details here, this concept is not nearly as speculative as it may sound. It actually requires the manifestation of only a single type of energy particle $[31,32]$.

\subsection{Gravitational Landscape}

Redefining the gravitational landscape: The boundary between the tetrahedral and octahedral domains may be described this way: On one side of the invisible dividing boundary, aether and embedded galaxies flow towards one gravitational center; beyond it, they flow towards another. As one astrophysicist aptly described it, "It's like water dividing at a watershed, where it flows either to the left or right of a height of land." So says Brent Tully, an astronomer at the University of Hawaii in Honolulu, who has charted the motions of galaxies to infer the gravitational landscape of the local Universe [33].

No doubt, the DSSU represents a profound redefining of the gravitational landscape. It is a universe consisting of a dense and infinite packing of cosmic-scale tetrahedral and octahedral gravity cells — perpetually self-sustaining. It is a universe of unified-gravity domains; and, on the large scale, nothing more.

\subsection{Source of Kinetic Energy}

One discovery, the cause of ellipticity, has led to another - the underlying source of grand-scale kinetic energy. We have a scenario (as has been presented in this paper) in which comotion molds the shape of galaxies. But note carefully, within a comoving environment this comotion does not represent kinetic energy. A string of co-drifting galaxies along $r$ have no kinetic energy with respect to each other and certainly not with respect to the space medium. However, when comotion ends, the motion of the now elliptically-shaped galaxy does represent kinetic energy. By virtue of having entered a cluster or having crossed into another subdomain, comotion becomes kinetic-energy motion. (While crossover into a subdomain is a common occurrence, the rule still holds that structures never venture outside their original nodal gravity domain.) In the transition from comotion to intrinsic motion the structure (and its component objects) acquires veritable kinetic energy. This happens when the structure enters the galaxy cluster neighborhood; and it happens when the structure crosses into a subdomain within a tetrahedral or octahedral gravity cell.

In a remarkable integration of ideas of galaxy evolution, within this transition of comotion to intrinsic motion and kinetic energy lies the key to the subsequent formation of spiral galaxies. Here is the key to the acquisition of large-scale angular momentum. When structures from opposing sub-domains meet, the result is a spectacular spectrum of morphologies - a catalogue of stellar structures - to delight astronomers and confound astrophysicists.

\subsection{Cosmology Implication}

The DSSU cosmology is based on (i) the Edwin Hubble discovery of the relationship between redshifted light and cosmic distance; (ii) the fact that medium expansion causes spectral redshifting; and (iii) the application of the velocity differential theory of cosmic redshift - a theory that proves redshift can occur in regions where the space medium expands as well as where it contracts [9].

The cosmic redshift is evidence of a dual dynamic space medium. The profound prediction is that the universe is divided into regions with expanding aether and regions with contracting aether. Our world is a stable cellular cosmos.

Conventional (i.e., universal-expansion) cosmology is based on (i) the same discovery made by Edwin Hubble of the redshifted-cosmic-distance relationship; (ii) the same fact that medium expansion causes photons to stretch; and (iii) an unscientific extrapolation of the application of the latter property - an unrestrained extrapolation of "space" expansion. The outrageously speculative result is an exploding universe! An unavoidable backwards-in-time extrapolation (a retrodiction) then leads to a big-bang genesis event.

Of particular interest is the prediction relating to the 
morphology of galaxy structures. And here it may be flatly and relevantly stated that the Expanding universe does not, and cannot, predict Elliptical galaxies. Within an expanding universe (even an accelerating expanding universe) ellipticity cannot be explained.

What this paper has shown is that the universe's cellularity, with its dual-dynamic aether, is essential for explaining the formation and ellipticity of galaxies (as well as the potential for subsequent galactic interaction).

We have here a mechanism that predicts, we have a mechanism that can be understood, we have a mechanism that explains galactic ellipticity.

\section{Appendix}

\section{Basic Aether-Inflow Equation}

Consider a spherical gravitating object, having mass $M$ and radius $R$, situated at-rest within a stationary aether medium. The aether velocity field, surrounding the object, may be found from Newtonian physics as follows: A small test-mass is resting at some arbitrary distance $r$ from the center of mass $M$. See Fig. A1. The small mass, designated as $m$, is "experiencing" a force, in accordance with Newton's Law of Gravity:

$$
F_{\text {gravity }}=-G M m / r^{2}, \text { where } M>>m \text { and } r>R .
$$

From Newton's $2^{\text {nd }}$ Law of Motion, a force is defined as $F=$ (mass) $\times($ acceleration $)$, so that

$$
m a=-G M m / r^{2} .
$$

Although at-rest in the frame of the sphere, the test mass is being subjected to acceleration; and whenever there is acceleration there must be a velocity (the velocity that is undergoing change). This velocity is found by replacing the acceleration with its definition, $a=d v / d t$ :

$$
\frac{d v}{d t}=\frac{d v}{d r} \frac{d r}{d t}=-\frac{G M}{r^{2}}
$$

which (after replacing $d r / d t$ with its identity $v$ ) may be integrated and solved for the velocity.

$$
\begin{aligned}
\int v d v & =-\int \frac{G M}{r^{2}} d r, \\
\frac{v^{2}}{2}=\frac{G M}{r}+C, \text { where } C & =0 \text { since } v=0 \text { when } r=\infty, \\
v^{2} & =\frac{2 G M}{r} .
\end{aligned}
$$

Although the test mass (being "stationary" in the M's reference-frame) clearly has no accelerating and no speed with respect to the gravitating body, it nevertheless is in motion. The test mass does have a speed with respect to the aether medium. The $v$ in the equation represents the relative speed between the test mass and aether.

$$
v= \pm \sqrt{2 G M / r}
$$

where $G$ is the gravitational constant and $r$ is the radial distance (from the center of the mass $M$ ) to any position of interest, at the surface of $M$, or external to $M$. The equation has two solutions. The positive solution expresses the "upward" motion of the test mass through the aether (in the positive radial direction). The negative solution represents the aether flow velocity (in the negative radial direction) streaming past the test mass - at the particular radial location specified by $r$.

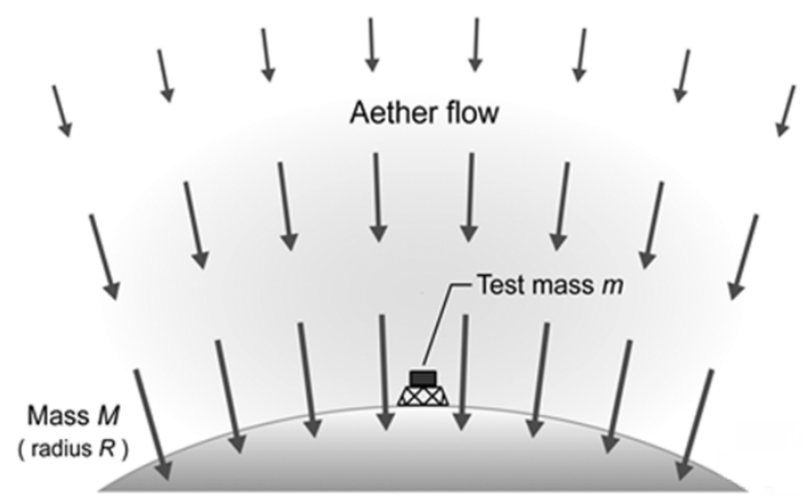

Figure A1. Aether streams and accelerates towards and into the large mass. The test-mass "experiences" the inflow acceleration as a gravity effect, and "experiences" the inflow speed as a radial component of absolute (aether-referenced) motion according to the formula, $\sqrt{2 G M / r}$.

On the cosmic scale, the above equation approximates the aether flow into a nodal galaxy cluster (mass $M_{C L}$ ):

$$
v_{\text {aetherflow }}=-\sqrt{2 G M \mathrm{CL} / r},
$$

where $r \geq$ (radius of cluster "surface").

\section{References}

[1] E. Hubble, Proc. Natl. Acad. Sci. USA 15, 168-173 (1929). (http://www.pnas.org/site/misc/Hubble.pdf)

[2] ScienceDaily, "Making stars: How cosmic dust and gas shape galaxy evolution," ScienceDaily, 2010 Nov 10. (http://www.sciencedaily.com/releases/2010/11/101122172012 .htm)

[3] S. Weinberg, Dreams of a Final Theory (Pantheon Books, NY, 1992) p239.

[4] R. Penrose, The Emperor's New Mind (Oxford University Press, New York, 1990) p328.

[5] Britannica, Vol.16, 15th Ed (Encyclopedia Britannica Inc., Chicago, 1991) p787.

[6] C. Ranzan, "The story of gravity and Lambda - How the theory of Heraclitus solved the dark matter mystery," Physics Essays Vol.23, No.1, p75-87 (2010).

[7] C. Ranzan, "The processes of gravitation -The cause and mechanism of gravitation," J. Mod. Phys. Appl. Vol.2014:3 (2014). (http://scik.org/index.php/jmpa/article/view/1138) 
[8] C. Ranzan, "Cosmic Redshift in the Nonexpanding Cellular Universe: Velocity-Differential theory of Cosmic Redshift," American Journal of Astronomy \& Astrophysics (AJAA), Vol.2, No.5, p47-60 (2014). (Doi: 10.11648/j.ajaa.20140205.11)

[9] C. Ranzan, Guide to the Construction of the Natural Universe (DSSU Research, Niagara Falls, Canada, 2014).

[10] ESO-CERN-ESA Symposium on Astronomy, Cosmology and Fundamental Physics, March 4-7, 2002. Garching bei München, Germany. (http://www.eso.org/sci/meetings/symp2002/)

[11] M. I. Scrimgeour et al., "The WiggleZ Dark Energy Survey: the transition to large-scale cosmic homogeneity," Monthly Notices of the Royal Astronomical Society, 2012; 425 (1): 116. (doi: 10.1111/j.1365-2966.2012.21402.x)

[12] P. Pearce, Structure in Nature Is a Strategy for Design (The MIT Press, Cambridge, Massachusetts, 1990) p42.

[13] J. P. Henry, U. G. Briel, H. Böhringer, "The evolution of galaxy clusters," Scientific American (Dec 1998).

[14] Space Telescope Science Institute. "Monster 'El Gordo' galaxy cluster is bigger than thought," ScienceDaily, 2014 April 3. (www.sciencedaily.com/releases/2014/04/140403141831.htm)

[15] B. Keel, "The Extragalactic Distance Scale," Web article (2003). (http://www.astr.ua.edu/keel/galaxies/distance.html)

[16] Kempner et al., "Chandra observations of A85: Merger of the South Subcluster," ApJ, 579, 236, (2002). (http://adsabs.harvard.edu/abs/2002ApJ...579..236K)

[17] The Cambridge Encyclopaedia of Astronomy. S. Mitton, Ed. (Crown Publishers, Inc., New York, N.Y., 1977) p331.

[18] T. T. Arny, Explorations: an Introduction to Astronomy, 2nd Ed (McGraw Hill, New York, N.Y., 2000) p480 (emphasis added).

[19] N. Wolchover, "Fluid tests hint at concrete quantum reality," Quanta Magazine, (2014 June 24). (https://www.quantamagazine.org/20140624-fluid-tests-hint-at -concrete-quantum-reality/)

[20] B. R. Greene, The Fabric of the Cosmos, Space, Time, and the Texture of Reality (Vintage Books, New York, 2005) p335.

[21] C. Ranzan, "The fundamental process of energy -A qualitative unification of energy, mass, and gravity," Infinite Energy Issue \#113 (Jan/Feb 2014) \& \#114 (Mar/Apr 2014). (http://www.infinite-energy.com/iemagazine/issue113/index.ht $\mathrm{ml})$ (http://www.infinite-energy.com/iemagazine/issue114/index.ht $\mathrm{ml})$

[22] T. T. Arny, Explorations: an Introduction to Astronomy, 2nd Ed. (McGraw Hill, New York, N.Y., 2000) p435.

[23] C. S. Powell, "Astronomy in the dark," Scientific American, (April 1995) p22.

[24] ScienceDaily, "Galaxies in the early universe mature beyond their years." ScienceDaily, 2013 March 10. (http://www.sciencedaily.com/releases/2014/03/14031021391 0.htm)

[25] S. L. Finkelstein et al., "A galaxy rapidly forming stars 700 million years after the Big Bang at redshift 7.51," Nature (2013) doi: $10.1038 /$ nature 12657

[26] A. P. Fairall, G. G. C. Palumbo, G. Vettolani, G. Kauffmann, A. Jones, G. Baiesi-Pillastrini, "Large-Scale Structure of the Universe ...," Mon. Not. R. astr. Soc. (1990) 247, Short Communication, p21-25.

[27] International Centre for Radio Astronomy Research (ICRAR). "Weirdness in cosmic web of the universe: Faint strings of galaxies in 'empty' space arranged in way never before seen," ScienceDaily, $2014 \quad$ March 10. (www.sciencedaily.com/releases/2014/03/140310090612.htm)

[28] S. D. Landy, "Mapping the Universe," Scientific American (June 1999) p38.

[29] G. Bothun, "Beyond the Hubble Sequence," Sky and Telescope (April 2000).

[30] Time-Life editors, Chap. "Galactic Conundrums," in Cosmic Mysteries (Time-Life Books, 1982) p70.

[31] C. Ranzan, "The Dynamic Steady State Universe," Physics Essays Vol.27, No.2, p286-315 (2014). (doi: http://dx.doi.org/10.4006/0836-1398-27.2.286)

[32] C. Ranzan, Guide to the Construction of the Natural Universe (DSSU Research, Niagara Falls, Canada, 2014) p82.

[33] E. Gibney, "Earth's new address: 'Solar System, Milky Way, Laniakea'," Nature, 2014 Sept. 3, Web article. (http://www.nature.com/news/earth-s-new-address-solar-syste m-milky-way-laniakea-1.15819). 